\title{
Modeling of flow stress in orthogonal micro-cutting process based on strain gradient plasticity theory
}

\author{
Jihua Wu Z Zhanqiang Liu
}

Received: 12 December 2008 / Accepted: 31 March 2009/Published online: 24 April 2009

(C) The Author(s) 2009. This article is published with open access at Springerlink.com

\begin{abstract}
The rapidly increasing demand for miniature components machining processes has drawn more attention to micro-machining research. Flow stress has always been a significant base for analyzing plastic deformation in machining processes. However, few studies have been conducted to predict accurately the material flow stress in the micro-cutting processes. In order to describe size effect in micro-cutting, this paper discusses the development of a circular primary deformation zone model, calculates the strain gradient in the primary zone, and presents a new flow stress model based on the theory of strain gradient plasticity. First, a series of orthogonal cutting experiments are performed and flow stress is calculated from the experiment data. Results from the proposed model have been successfully validated with experimentally determined results. It shows that the flow stress in micro-cutting is influenced greatly by the feed rate and the cutting edge radius.
\end{abstract}

Keywords Micro-cutting · Stain gradient · Flow stress · Dislocation density

\section{Introduction}

The marked increase in demand for miniaturized products in various fields such as medical, telecommunication, avionics, biotechnology, and electronics demands that the

\footnotetext{
J. Wu $(\bowtie) \cdot$ Z. Liu

School of Mechanical Engineering, Shandong University, 250061 Jinan, People's Republic of China

e-mail:wujihua@mail.sdu.edu.cn

Z. Liu

e-mail: melius@sdu.edu.cn
}

micro-machining processes develop rapidly. Micro-cutting allows the creation of miniaturized products that are more compact, reliable, efficient, safe, environmentally friendly, and cost competitive, which has become an important enabling technology comparing to lithographic, etching, and laser techniques. Many studies of micro-cutting mechanics have received increased emphasis.

Both the finite element method (FEM) and analytical modeling require flow stress data of the workpiece material to analyze the deformation during the metal cutting process. Flow stress is always a significant baseline for analyzing cutting processes. Many research studies have been done on macro-cutting using the flow stress model. Merchant assumed that the shear flow stress in metal cutting was equal to that obtained from conventional tensile tests [1]. However, the intense circumstance in metal cutting results in the mechanical material behavior is far beyond from that encountered in conventional material tests.

Several classic plasticity models including the early power law model, the Johnson-Cook (JC) model, the Usui model mechanical threshold stress model, the Zerilli model, etc. have been developed to relate flow stress to plastic strain, strain rate, and/or temperature. These models have been widely employed in macro-cutting processes [2].

Researchers have tried to demonstrate that the flow stress models in macro scale referred to above could be applied to the micro-cutting processes. Sathyan used the JohnsonCook model to simulate ductile fracture in micro-cutting of Al2024-T3 [3]. Woon used the Johnson-Cook model to investigate the effect of tool edge radius and chip formation behavior in micro-machining of AISI 4340 steel using finite microelement analysis. Simoneau illustrated the effect of material microstructure on chip formation during microscale cutting using the strain rate-dependent Johnson-Cook model [5]. 
Classical theories of plasticity possess no internal length scale. As a result, they have at least two major drawbacks:

They cannot model the well-known size effect observed in micro-cutting processes where the specific cutting energy/force increases non-linearly with a decrease in uncut chip thickness.

They cannot model materials undergoing inhomogeneous plastic flow in micro-cutting processes or model the occurrence of strains localization in the primary deformation zone and chips.

These drawbacks have initiated the application of strain gradient plasticity theory in micro-cutting. Laheurte quantified energy parameters involved in the cutting process based on the strain gradient theory with couple stresses [6]. Joshi modeled size effect in the parallel-sided primary deformation zone by using a sharp cutting tool in machining utilizing the strain gradient plasticity theory [7] without considering the effect of cutting edge radius. However, the cutting edge radius should not be neglected when the undeformed chip thickness is comparable in size to the cutting edge radius. Liu developed a coupled thermo-mechanical finite element model capable of accurately predicting the size effect in specific cutting energy formulation incorporating strain gradient plasticity to simulate orthogonal micro-cutting processes [8].

Because the FEM method is complicated and tedious, few researches are engaged in an accurate analysis model of material flow stress in the micro-cutting processes until now.

The objective of this paper is to model the flow stress that emerges in the deformation phenomena in microcutting based on the strain gradient plasticity theory. Orthogonal cutting experiments are performed to validate the proposed flow stress model.

\section{Flow stress modeling based on strain gradient plasticity theory}

\subsection{Taylor dislocation model}

The Taylor dislocation model defines the shear flow stress $\tau$ in terms of the dislocation density.

$\tau=\alpha_{c} G b \sqrt{\rho_{\text {total }}}$

where $\alpha_{c}$ is constant to be taken as 0.5 [9], $G$ is the material shear modulus, $b$ is the magnitude of Burgers vector, and $\rho_{\text {total }}$ is total dislocation density.

The total dislocation density $\rho_{\text {total }}$ is composed of the density of statistically stored dislocations and the density of geometrically necessary dislocations.

$\rho_{\mathrm{total}}=\rho_{\mathrm{SSD}}+\rho_{\mathrm{GND}}$
$\rho_{\mathrm{SSD}}$ is the density of statistically stored dislocations which accumulate by trapping each other in a random way, and $\rho_{\mathrm{GND}}$ is the density of geometrically necessary dislocations, which are required for compatible deformation of various parts of the non-uniformly deformed material.

The tensile flow stress $\sigma$ is related to shear flow stress $\tau$ by:

$\sigma=M \tau$

where $M$ is constant and $M=\sqrt{3}$ for isotropic materials.

The density of statistically stored dislocation $\rho_{\mathrm{SSD}}$ can be determined from the uniaxial stress-strain law in the absence of strain gradient effects as,

$\sigma_{0}=M \alpha_{c} G b \sqrt{\rho_{\mathrm{SSD}}}$

where $\sigma_{0}$ is the reference stress in uniaxial tension.

Based on Eqs. (1)-(4), a flow stress equation accounting for the effect of geometrically necessary dislocations can be written as,

$$
\begin{aligned}
\sigma & =M \alpha_{c} G b \sqrt{\rho_{\mathrm{SSD}}+\rho_{\mathrm{GND}}}=M \alpha_{c} G b \sqrt{\rho_{\mathrm{SSD}}} \sqrt{1+\frac{\rho_{\mathrm{GND}}}{\rho_{\mathrm{SSD}}}} \\
& =\sigma_{0} \sqrt{1+\frac{\rho_{\mathrm{GND}}}{\rho_{\mathrm{SSD}}}}
\end{aligned}
$$

The density of geometrically necessary dislocations, can be related to the effective strain gradient $\eta$ as,

$\rho_{\mathrm{GND}}=\frac{\bar{r} \eta}{b}$

where $\bar{r}$ is Nye factor to reflect the effect of crystallography on the distribution of GNDs, which is around 2 for the polycrystalline material [10].

Substituting Eq. (6) into Eq. (5) and is rearranged as:

$\sigma=\sigma_{0} \sqrt{1+l \eta}$

where $l$ is the intrinsic material length scale so as to balance the dimensions of strains and strain gradients and can be given as,

$l=\frac{M^{2} \bar{r} \alpha^{2} G^{2} b}{\sigma_{0}^{2}}$

The intrinsic material length $l$ represents a natural combination of the effect of elasticity, plasticity, and dislocation structure in terms of the shear modulus, Burgers vector $b$, and coefficient $\alpha_{c}$ in the Taylor model. The material length scale of AISI 1045 is $27.6 \mu \mathrm{m}$ reported by Kopalinsky [11].

\subsection{Evaluation strain gradient in micro-cutting}

The strain gradient $\eta$ should be obtained in order to determine the flow stress model according to Eq. (7). 
Procedures of modeling of flow stress in micro-cutting involves: (1) determination of the geometry of the primary zone; (2) evaluation of the strain gradient and density of dislocations; and (3) evaluation of flow stress.

\subsubsection{Determination of geometry of the primary zone}

Many geometrical shapes have been proposed to describe the deformation zone in macro-cutting such as shear plane [1], parallel-sided shear zone [12], or curved shear zone [13]. Cutting tools commonly used in machining operations are never ideally sharp but always have some bluntness. The bluntness can often be approximated as a circular radius between the flank and the rake faces of the tool. Recently, many researchers have found that the shape of the primary shear zone within micro-cutting processes was circular vicinity ahead of the circular edge by FEM method $[4,14]$. Assuming no material elastic recovery of machined surface, the circular primary deformation zone parallel to the cutting edge model is therefore proposed.

\subsubsection{Evaluation of the strain gradient in the primary deformation zone}

Researchers assume that macro orthogonal cutting is similar to wedge indentation for the reason that the flow lines formed by indentation in experiments look like a consequence of the shear deformation occurring along the primary shear zone, as is shown in Fig. 1 [15].

Therefore, one can use the micro-indentation model of GNDs developed by Nix [9], Qu [16], and Al-Rub [17] to determine the density of GNDs evolved under spherical indenter in micro-indentation. If a hard transverse cylinder slides on a soft surface, the scenario would more closely represent machining with a rounded tool edge. The cutting width $w$ is the height of the cylinder and the cutting edge radius $r_{\mathrm{e}}$ is the cylindrical radius. Non-uniform plastic deformation occurs in the circular primary deformation zone and elastic deformation occurs outside of the deformation zone. One can assume that the density of GNDs is integrated along the circular cutting edge, and the

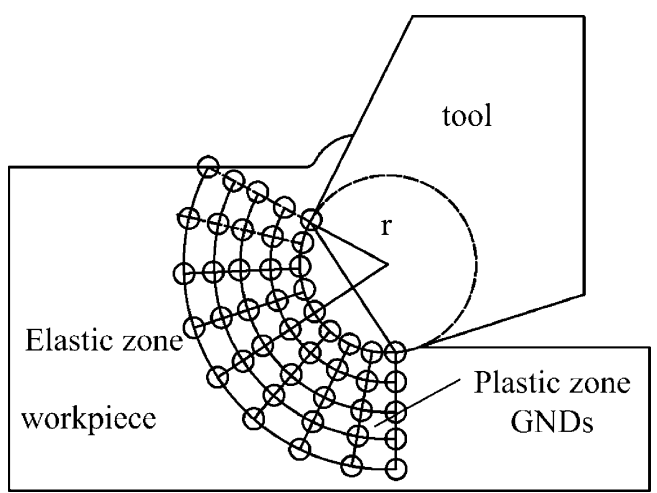

Fig. 2 Micro-cutting model based on dislocation mechanics

cutting edge is accommodated by rectangle dislocation loops of GNDs with Burgers vector normal to the plane of the machined surface as shown in Figs. 2 and 3.

In the model developed by Nix [9], the density of GNDs is derived from the total line length $\lambda$ of dislocation loops necessary to form the shape of tool edge. The schematic diagram of the circular primary zone is shown in Fig. 4. The total length of the dislocation loops is then divided by the part of column $V$ by the contact length $a$. The cylinder volume $V$ of the contact length $a$ is given by:

$V=\left(\frac{\pi r_{\mathrm{e}}^{2}(90+\gamma+\alpha)}{180}-a r_{\mathrm{e}}\left(1-\cos \left(\frac{\pi}{4}+\frac{\gamma+\alpha}{2}\right)\right)\right) w$

where $\gamma$ is the effective rake angle of the tool and $\alpha$ is the clearance angle. When machining at very low values of undeformed chip thickness, the effective rake angle as shown in Fig. 5 changes and can be given by Eq. (10):

$$
\gamma= \begin{cases}\sin ^{-1}\left(\frac{t}{r_{\mathrm{e}}}-1\right) t & \leq r_{\mathrm{e}} \\ \gamma_{\mathrm{n}} & t>r_{\mathrm{e}}\end{cases}
$$

where $\gamma_{\mathrm{n}}$ is the nominal rake angle of the tool and $t$ is the undeformed chip thickness.

The contact length $a$ can be calculated by:

$$
a=r_{\mathrm{e}} \sin \left(\frac{\pi}{4}+\frac{\gamma+\alpha}{2}\right)
$$

Fig. 1 Flow lines observed in experiments

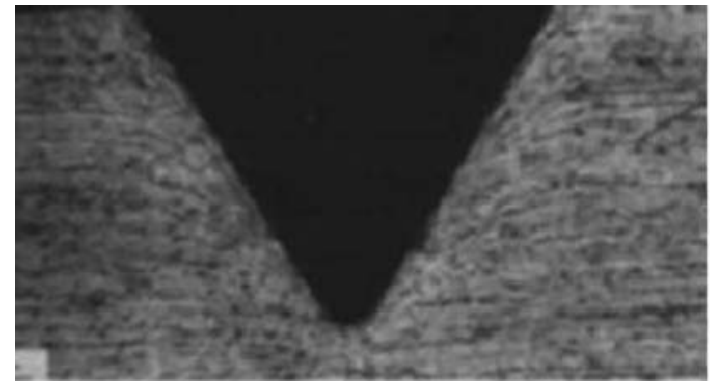

(a) Indentation

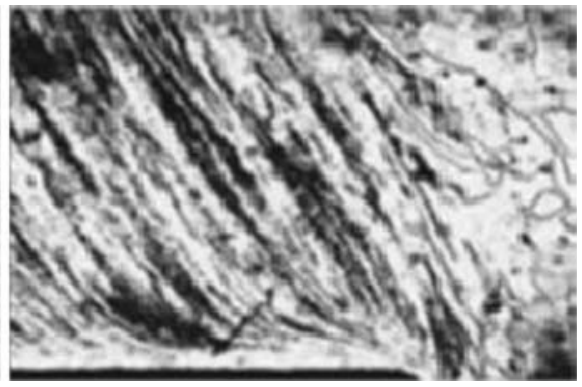

(b) Machining 
We assume that the individual dislocation loops of GNDs are spaced equally along the cutting tool edge surface.

$\frac{b}{L_{\mathrm{G}}}=\frac{h}{a}$

where $L_{\mathrm{G}}$ is the mean spacing between individual slip steps on the edge surface corresponding to the GND loops. $h$ is the ploughing depth, which can be given by:

$h=r_{\mathrm{e}}\left(1-\cos \left(\frac{\pi}{4}+\frac{\alpha+\gamma}{2}\right)\right)$

If $\lambda$ is the total length of the injected loops, then the length of the injected loops between the $x$ and $x+\Delta x$ as shown in Fig. 3 can be written by:

$\mathrm{d} \lambda=(2 \mathrm{w}+4 \mathrm{y}) \frac{\mathrm{dx}}{\mathrm{L}_{\mathrm{G}}}$
Integrating from 0 to the contact length $a$ gives the total length of GND loops as follows:

$\lambda=\int_{0}^{a}(2 w+4 y) \frac{\mathrm{dx}}{L_{\mathrm{G}}}=\frac{h}{b a} \int_{0}^{a}(2 w+4 y) \mathrm{dx}$

where $y=\sqrt{r_{\mathrm{e}}^{2}-x^{2}}$.

So one can calculate the total length of the GND loops as follows:

$$
\begin{aligned}
\lambda=r_{\mathrm{e}} & \left(1-\cos \left(\frac{\pi}{4}+\frac{\alpha+\gamma}{2}\right)\right) \\
& \left(\frac{2 w}{b}+\frac{r_{\mathrm{e}}\left(\frac{\pi}{4}+\frac{\gamma+\alpha}{2}\right)}{b \sin \left(\frac{\pi}{4}+\frac{\gamma+\alpha}{2}\right)}-\frac{r_{\mathrm{e}} \cos \left(\frac{\pi}{4}+\frac{\gamma+\alpha}{2}\right)}{2 b}\right)
\end{aligned}
$$

Therefore, the density of GNDs becomes,

$\rho_{\mathrm{GND}}=\frac{\lambda}{V}=\frac{\left(1-\cos \left(\frac{\pi}{4}+\frac{\alpha+\gamma}{2}\right)\right)\left(\frac{2 w}{b}+\frac{r_{\mathrm{e}}\left(\frac{\pi}{4}+\frac{\gamma+\alpha}{2}\right)}{b \sin \left(\frac{\pi}{4}+\frac{\gamma+\alpha}{2}\right)}-\frac{r_{\mathrm{e}} \cos \left(\frac{\pi}{4}+\frac{\gamma+\alpha}{2}\right)}{2 b}\right)}{\left(\frac{\pi r_{\mathrm{e}}(90+\gamma+\alpha)}{180}-r_{\mathrm{e}} \sin \left(\frac{\pi}{4}+\frac{\gamma+\alpha}{2}\right)\left(1-\cos \left(\frac{\pi}{4}+\frac{\gamma+\alpha}{2}\right)\right)\right) w}$

And the effective strain gradient can be calculated using

Eq. (6).

$\eta=\frac{b \rho_{\mathrm{GND}}}{2}=\frac{b}{2} \frac{\left(1-\cos \left(\frac{\pi}{4}+\frac{\alpha+\gamma}{2}\right)\right)\left(\frac{2 w}{b}+\frac{r_{\mathrm{e}}\left(\frac{\pi}{4}+\frac{\gamma+\alpha}{2}\right)}{b \sin \left(\frac{\pi}{4}+\frac{\gamma+\alpha}{2}\right)}-\frac{r_{\mathrm{e}} \cos \left(\frac{\pi}{4}+\frac{\gamma+\alpha}{2}\right)}{2 b}\right)}{\left(\frac{\pi r_{\mathrm{e}}(90+\gamma+\alpha)}{180}-r_{\mathrm{e}} \sin \left(\frac{\pi}{4}+\frac{\gamma+\alpha}{2}\right)\left(1-\cos \left(\frac{\pi}{4}+\frac{\gamma+\alpha}{2}\right)\right)\right) w}$

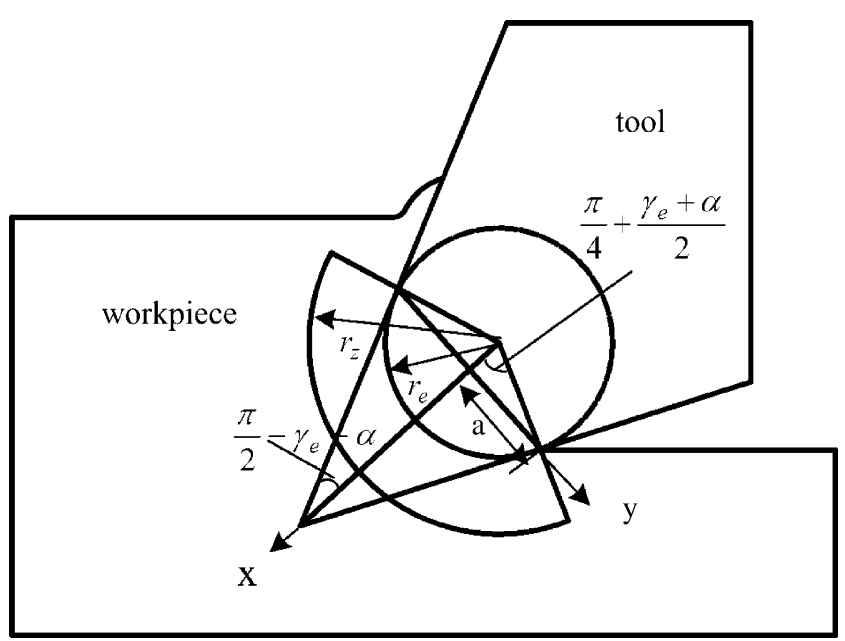

Fig. 3 Schematic diagram of circular primary zone
Substituting Eq. (18) into Eq. (7), one can calculate the flow stress in micro-cutting.

Liu [8] and Woon [4] revealed that the plastic zone ahead of tool tip region increased with the reduction of $t / r_{\mathrm{e}}$. Johnson [18] estimated the size of the plastic zone from contact radius and the radius $r_{\mathrm{e}}$ of spherical indenter based on the strain gradient plasticity theory. According to Johnson's model, the radius of circular plastic zone ahead of tool tip region $r_{z}$ is calculated by:

$$
\begin{aligned}
r_{z} & =\frac{r_{\mathrm{e}} h^{2}}{2 a^{2}}+r_{\mathrm{e}}=r_{\mathrm{e}}\left(1+\frac{h^{2}}{2 a^{2}}\right) \\
& =r_{\mathrm{e}}\left(1+\frac{\left(1-\cos \left(\frac{\pi}{4}+\frac{\gamma+\alpha}{2}\right)\right)^{2}}{2 \sin ^{2}\left(\frac{\pi}{4}+\frac{\gamma+\alpha}{2}\right)}\right)
\end{aligned}
$$




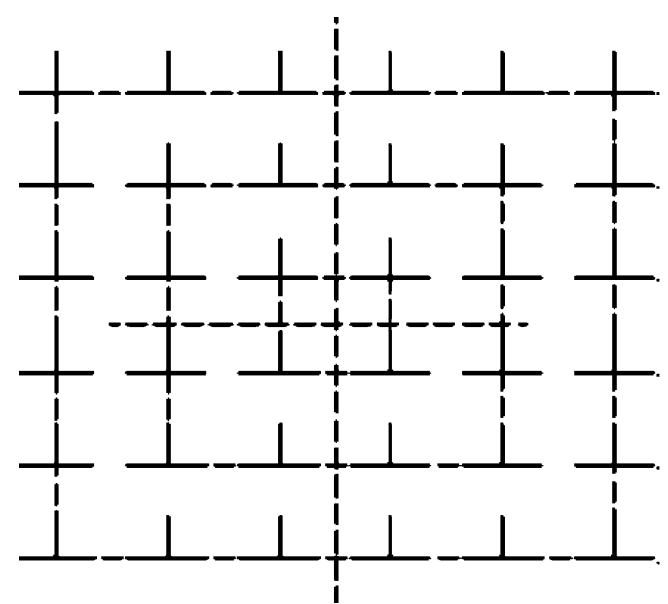

Fig. 4 Arrangement of GNDs in the $x$ direction

\section{Experimental verification}

The experimental investigation was undertaken on a ACEV500 milling machine. The tool was mounted directly on Kistler Type 9275A, three-component piezoelectric force dynamometer whose resolution is $1 \mathrm{mN}$. The cutting edge was orthogonal to the workpiece. The cutting forces $F_{\mathrm{c}}$ and thrust forces $F_{\mathrm{t}}$ in the orthogonal cutting were measured. The configuration details of the experimental setup were shown in Fig. 6. AISI 1045 was chosen as the workpiece material. The workpiece was in the form of tube of $1-\mathrm{mm}$ wall thickness; the geometry of the workpiece is shown in Fig. 7. The experimental details were given in Table 1.

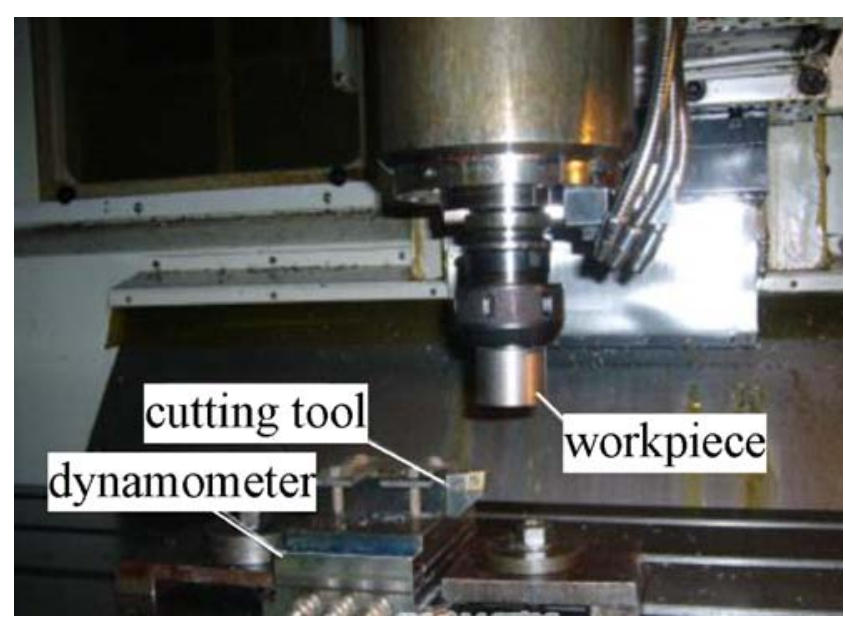

Fig. 6 Schematic of the experimental setup

\section{Results and discussion}

\subsection{Experiment data validation}

The average flow stress can be obtained from the cutting and thrust forces using Eq. (20).

$\sigma=\frac{\left(F_{\mathrm{c}} \sin \phi+F_{\mathrm{t}} \cos \phi\right) \sin \phi}{t w}$

The prediction of shear angle $\varphi$ by Merchant's equation can be obtained from Eq. (21) and friction angle

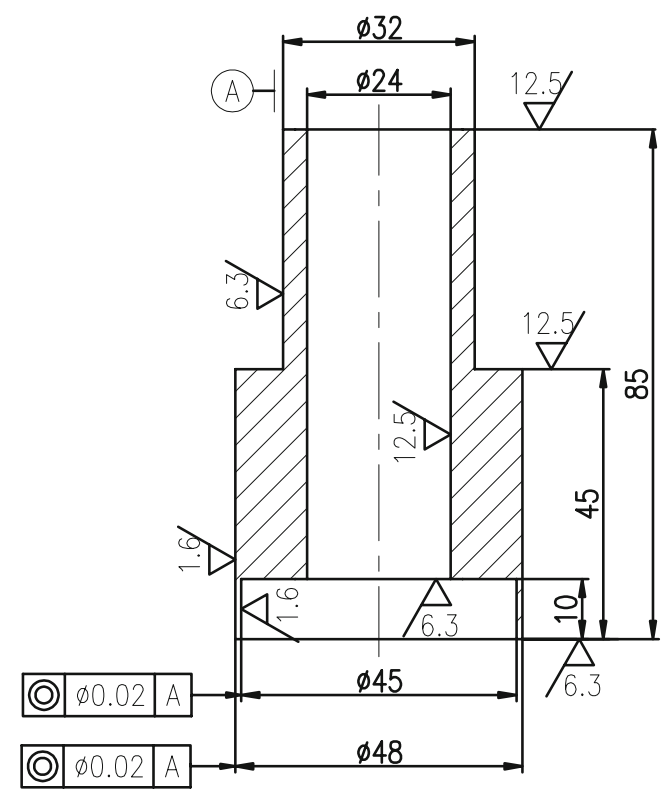

Fig. 7 Geometry of workpiece

Fig. 5 Effective rake angle in micro-cutting 
Table 1 Cutting conditions and tool geometries

\begin{tabular}{ll}
\hline Tool & \\
\hline $\begin{array}{l}\text { Tool material } \\
\text { Tool geometry }\end{array}$ & $\begin{array}{l}\text { Kenna KC9315 } \\
\text { CNMG120408UN } 0^{\circ} \text { rake angle, } \\
5^{\circ} \text { clearance angle }\end{array}$ \\
$\begin{array}{l}\text { Tool edge radius }(\mu \mathrm{m}) \\
\text { Workpiece material }\end{array}$ & $\begin{array}{l}\text { AISI } 1045 \\
\text { Cutting conditions }\end{array}$ \\
$\begin{array}{l}\text { Cutting speed }(\mathrm{m} / \mathrm{min}) \\
\text { Undeformed chip } \\
\text { thickness }(\mu \mathrm{m} / \mathrm{r})\end{array}$ & $15.7,39.25,62.8,78.5,117.75,141.3$ \\
Cutting width $(\mathrm{mm})$ & $1,2,3,4,5,6,7,8,9,10$ \\
\hline
\end{tabular}

$\beta$ can be obtained from Eq. (22) in force equilibrium condition.

$\phi=\frac{\pi}{4}+\frac{\alpha}{2}-\frac{\beta}{2}$

$\beta=\tan ^{-1}\left(\frac{F_{\mathrm{t}}+F_{\mathrm{c}} \tan \alpha}{F_{\mathrm{c}}-F_{\mathrm{t}} \tan \alpha}\right)$

The errors between experimental measurement and model prediction were calculated using Eq. (23) and can be found in Table 2. It can be seen from Table 2 that an absolute average percentage error of $11.72 \%$ for flow stress at cutting speed of $15.8 \mathrm{~m} / \mathrm{min}$ and an absolute average percentage error of $25.2 \%$ at cutting speed of $141.3 \mathrm{~m} / \mathrm{min}$ are obtained from orthogonal cutting tests.

$$
\begin{aligned}
\text { Error }= & \frac{\mid \text { experimental value }- \text { simulated value } \mid}{\text { experimental value }} \\
& \times 100 \%
\end{aligned}
$$

\subsection{Effect of feed rate}

Figure 8 shows the effect of the undeformed chip thickness (feed rate in orthogonal cutting) on the flow stresses at the cutting speed of $15.8 \mathrm{~m} / \mathrm{min}$. As the feed rate decreases, the flow stress increases, which is the so-called size effect.

Since strain gradients are very intense due to the storage of GNDs in micro-cutting, in this research, we can attribute the size effect to the appearance of plastic strain gradients. According to Eq. (19), the relation of strain gradient and

Table 2 Comparison between analytical and simulation values for flow stresses

\begin{tabular}{lccc}
\hline & $\begin{array}{l}\text { Effective normal } \\
\text { stress predicted } \\
\text { by model }\end{array}$ & $\begin{array}{l}\text { Effective normal } \\
\text { stress experiment }\end{array}$ & Error (\%) \\
\hline $15.8 \mathrm{~m} / \mathrm{min}$ & 358.96 & 406.60 & 11.72 \\
$141.3 \mathrm{~m} / \mathrm{min}$ & 951.45 & 761.16 & 25.2 \\
\hline
\end{tabular}

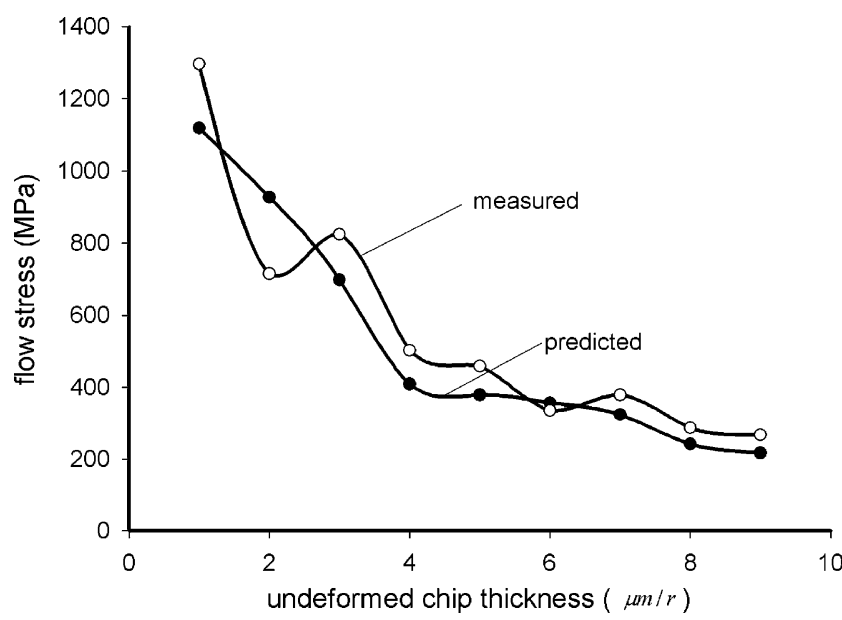

Fig. 8 Effect of the undeformed chip thickness on the flow stresses

effective rake angle calculated by the undeformed chip thickness has been built quantifiably to represent the influence of the feed rate on the flow stress in the microcutting process. The strain gradient enhances the flow stress with decrease of the feed rate.

\subsection{Effect of cutting velocity}

Figure 9 shows the effect of cutting velocity on the flow stresses when the feed rate is $8 \mu \mathrm{m} / \mathrm{r}$. It is seen that the flow stress predicted from the proposed model agrees well with the experimental data when the cutting velocity is lower than $78.5 \mathrm{~m} / \mathrm{min}$. A significant error exists when the cutting velocity exceeds the critical speed $78.5 \mathrm{~m} / \mathrm{min}$. This phenomenon can be explained by the strain rate effect. Eleiche [19] found that flow stress is very sensitive to strain rate. Three regions with different deformation mechanics are divided according to strain rate sensitivity. In a low strain rate range, the flow stress is dominated by dislocation density and hardly influenced by the strain

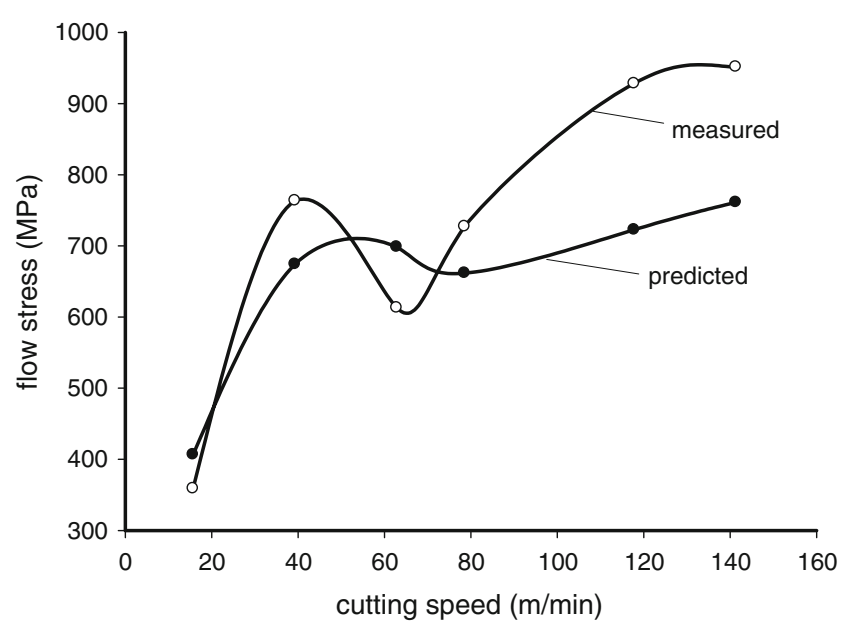

Fig. 9 Effect of the cutting velocity on the flow stress 
rate. However, during high speed ranges, the flow stress will be mainly influenced by the strain rate. The strain gradient plasticity theory does not work in such high speed ranges. So when the velocity exceeds a critical value, the strain gradient plasticity theory cannot predict the flow stress well.

\section{Conclusion}

In this paper, a flow stress model based on strain gradient plasticity theory has been proposed for the process of micro-cutting. The proposed model relates the flow stress in micro-cutting to tool geometry (edge radius, rake angle, and clearance angle) and cutting condition (undeformed chip thickness, cutting width). It is available to predict the size effect and size of the primary deformation zone.

A series of orthogonal cutting experiments were performed and flow stresses were calculated with experimental data. An absolute average percentage error of $11.72 \%$ for flow stress at cutting speed of $15.8 \mathrm{~m} / \mathrm{min}$ and an absolute average percentage error of $25.2 \%$ at cutting speed of $141.3 \mathrm{~m} / \mathrm{min}$ were obtained for orthogonal cutting tests.

The following conclusions can be drawn:

1. As the feed rate decreases, the flow stress increases.

2. The flow stresses are sensitive to strain rate. In a low strain rate range, the flow stress is dominated by dislocation density and hardly influenced by the strain rate. While the cutting velocity exceeds a critical value, the strain gradient plasticity cannot predict the flow stress well.

Acknowledgements This project is supported by National Basic Research Program of China (no. 2009CB724401), National Natural Science Foundation of China (through grants no. 50675122 and 50828501), Natural Science Foundation of Shandong Province, China (through grants no. Z2007F03 and 2007ZCB01518), and Excellent Postdoctoral Foundation of Shandong Province (through grant no. 200602008).

Open Access This article is distributed under the terms of the Creative Commons Attribution Noncommercial License which permits any noncommercial use, distribution, and reproduction in any medium, provided the original author(s) and source are credited.

\section{References}

1. Merchant ME (1945) Mechanics of the metal cutting process. Orthogonal cutting and type 2 chip. J Appl Phys 16(5):267-276. doi:10.1063/1.1707586

2. Partchapol S, Frank K, Taylan A (2004) Determination of flow stress for metal cutting simulation - a progress report. J Mater Process Technol 146:61-71. doi:10.1016/S0924-0136(03)00845-8

3. Subbiah S (2006) Some investigations of scaling effects in microcutting. Georgia Institute of Technology

4. Woon KS, Rahman M, Fang FZ, Neo KS, Liu K (2008) Investigations of tool edge radius effect in micromachining: a FEM simulation approach. J Mater Process Technol 195(13):204-211. doi:10.1016/j.jmatprotec.2007.04.137

5. Simoneau A, Ng E, Elbestawi MA (2007) Grain size and orientation effects when microcutting AISI 1045 steel. Ann CIRP 56:57-61. doi:10.1016/j.cirp.2007.05.016

6. Laheurte R, Cahuc O, Darnis P, Gerard A (2006) Behaviour law for cutting process. Int J Adv Manuf Technol 29:17-23. doi:10.1007/s00170-004-2498-4

7. Joshi SS (2004) An explanation for the size-effect in machining using strain gradient plasticity. J Manuf Sci E-T ASME 126:679685. doi:10.1115/1.1688375

8. Liu K (2005) Processes modeling of micro-cutting including strain gradient effects. Georgia Institute of Technology

9. Nix WD, Gao H (1998) Indentation size effects in crystalline materials: a law for strain gradient plasticity. J Mech Phys Solids 46(3):411-425. doi:10.1016/S0022-5096(97)00086-0

10. Arsenlis A, Parks DM (1999) Crystallographic aspects of geometrically-necessary and statically-stored dislocation density. Acta Mater 47:1597-1611. doi:10.1016/S1359-6454(99)00020-8

11. Kopalinsky EM, Oxley PLB (1984) Size effects in metal removal processes. Inst Phys Conf Ser n70:389-396

12. Oxley PLB (1961) Mechanics of metal cutting. Int J Mach Tool D R 1(1-2):89-97. doi:10.1016/0020-7357(61)90046-4

13. Fang N (2003) Slip-line modeling of machining with a roundededge tool-Part II: analysis of the size effect and the shear strainrate. J Mech Phys Solids 51(4):743-762. doi:10.1016/S0022-5096 (02)00061-3

14. Liang SY (2006) Mechanical machining and metrology at micro/ nano scale. Proc. SPIE 6280:1-8

15. Masuzawa T, Tönshoff HK (1997) Three-dimensional micromachining by machine tools. Ann CIRP 46(2):621-628

16. Qu S, Huang Y, Pharr GM, Hwang KC (2006) The indentation size effect in the spherical indentation of iridium: a study via the conventional theory of mechanism-based strain gradient plasticity. Int J Plast 22(7):1265-1286

17. Abu AR, Voyiadjis GZ (2004) Analytical and experimental determination of the material intrinsic length scale of strain gradient plasticity theory from micro- and nano-indentation experiments. Int J Plast 20(6):1139-1182

18. Johnson KL (1970) Correlation of indentation experiments. J Mech Phys Solids 18(2):115-126

19. Eleiche A, Campbell J (1976) Strain-rate effects during reverse torsional shear. Exp Mech 16(8):281-290 